\title{
NoGluten: Plataforma de apoyo a la celiaquía
}

\author{
NoGluten: Celiac Support Platform
}

Gonzalo Alvariño ${ }^{1}$, Manuela Cannella ${ }^{2}$, Facundo Laxalde ${ }^{3}$, Leandro Nuñez ${ }^{4}$, José Solsona ${ }^{5}$

Recibido: Febrero 2020

Aceptado: Noviembre 2019

Resumen.- El proyecto busca solucionar la falta de información centralizada y actualizada que hoy enfrentan personas con algún tipo de intolerancia al gluten. Actualmente en Uruguay, se estima que existen 30.000 celíacos diagnosticados, lo que lleva a que toda la comunidad relacionada con esta intolerancia, incluyendo familiares y amigos, aumente a un mínimo de 80.000 personas. NoGluten es una plataforma de apoyo colaborativa, cuyo objetivo es brindar respaldo y facilidades a esta comunidad, permitiendo escanear un producto con código de barras para comprobar si es apto para el consumo, disponer de un listado de productos avalados de manera instantánea y fidedigna, localizar sitios gastronómicos y proveedores de servicios, encontrar recetas, compartir experiencias de viajes, entre otros. Consiste en una aplicación móvil para dispositivos Android e iOS, y un sitio web responsive, para administradores y proveedores, donde los últimos pueden cargar sus productos, para promocionarlos mediante la aplicación. La plataforma está siendo utilizada por usuarios reales. El sitio web se encuentra publicado en http://www.noglutenapp.com/. La aplicación móvil también está disponible en ambas plataformas.

Android: https://play.google.com/store/apps/details?id=org.nativescript.NoGlutenApp\&hl=en iOS: https://apps.apple.com/uy/app/no-gluten/id1397633803?l=es

Palabras clave: Celíaco; Celiaquía; Gluten; Contaminación cruzada; Dieta libre de gluten.

Summary.- The project arises seeking to solve the lack of centralized and updated information that people with some type of gluten intolerance suffer today. Currently in Uruguay, it is estimated that there are about 30,000 diagnosed celiacs, which leads the entire community related to this intolerance, including family and friends, to a minimum of 80,000 people. NoGluten is a collaborative support platform, whose objective is to provide support and facilities to this community, allowing to scan a product with a barcode to check if it is suitable for consumption, have a list of guaranteed products instantly and reliably, locate Gastronomic sites and service providers, share travel experiences, among others. Consists of a mobile app for Android and IOS devices, and a web application, for administrators and suppliers, where they can upload their products and promote them through the app. The website is published at http://www.noglutenapp.com/. The mobile application is also available on both platforms. Android: https://play.google.com/store/apps/details?id=org.nativescript.NoGlutenApp\&hl=en iOS: https://apps.apple.com/uy/app/no-gluten/id1397633803?l=es

Keywords: Celiac; Celiac disease; Gluten; Cross contamination; Gluten free diet.

\footnotetext{
${ }^{1}$ Ing. en Sistemas, Universidad ORT Uruguay, galvarin91@gmail.com, ORCID iD: 0000-0003-2809-0821

${ }^{2}$ Ing. en Sistemas, Universidad ORT Uruguay, manucannella6@gmail.com, ORCID iD: 0000-0003-4709-6487

${ }^{3}$ Ing. en Sistemas, Universidad ORT Uruguay, facundolaxalde@gmail.com, ORCID iD: 0000-0002-9057-7924

${ }^{4}$ Ing. en Sistemas, Universidad ORT Uruguay, leanunes16@gmail.com, ORCID iD: 0000-0002-7395-5268

${ }^{5}$ Ing. en Sistemas, Universidad ORT Uruguay, josedusolsona@gmail.com, ORCID iD: 0000-0001-8626-9569
} 


\section{Introducción.-}

1.1. Celiaquía.- La enfermedad celíaca (EC), patología autoinmune, consiste en una intolerancia a las proteínas del gluten que se presenta en aquellas personas con vulnerabilidad genética para desarrollar la enfermedad [1-2]. Tras la ingesta se generan fragmentos proteicos, prolaminas, que a su vez se componen de gliadinas y gluteninas y resultan tóxicos para las personas celiacas [3]. En respuesta a la ingesta de gluten, el sistema inmune lesiona principalmente al aparato digestivo, fundamentalmente la mucosa del intestino delgado (órgano donde se realiza la absorción de los alimentos), comprometiendo la incorporación de un vasto conjunto de nutrientes. Esto determina un mal estado nutricional que compromete la salud general de quien lo padece [4].

Esta condición es de carácter permanente, se mantiene a lo largo de la vida y el tratamiento que estrictamente debe seguirse es "la dieta libre de gluten" que no sólo implica consumir alimentos libres de gluten sino también evitar la contaminación cruzada a partir de alimentos que contengan gluten. El sistema inmune reacciona ante cantidades que podríamos denominar ínfimas. (a modo de ejemplo, una persona celíaca no podrá consumir un "tuco" elaborado con ingredientes aptos si otra persona sumergió un trozo de pan para "probarlo" o utilizó para mezclar una cuchara que estuvo en contacto con el agua de la pasta elaborada con trigo). Si la persona celíaca adhiere a la dieta libre de gluten, la absorción de nutrientes se recupera y la morbi mortalidad es comparable a la población no celíaca [4-5].

1.2. Extendido uso del gluten.- Los cereales TACC son de muy amplio uso tanto en la comida elaborada en el hogar o restaurantes, como la de origen industrial. Es más, muchas veces su incorporación en distintas recetas busca las propiedades que el gluten aporta, tanto en masas como para el "espesado" de alimentos salados o dulces. Esta situación obliga a que los insumos y alimentos elaborados a ser consumidos por la persona celíaca deban tener una adecuada identificación como "libre de gluten"; con excepción de los alimentos exentos de gluten (frutas, verduras, carnes, arroz, etc; siempre y cuando no exista riesgo de contaminación cruzada) [4-6].

1.3. Falta de información consolidada y que responda a los principios de la usabilidad y la experiencia de usuario.- Afortunadamente, la oferta de insumos y alimentos libre de gluten es extendida y continúa en crecimiento. El problema radica en la carencia de un repositorio de datos actualizado y fácilmente accesible, que permita explotar el conjunto de posibilidades adecuado a la necesidad puntual del usuario. Una constante es la restricción que asume la persona celíaca por falta de información sobre el espectro de posibilidades que tiene delante [7-8].

Es atendiendo esta situación que se elaboró la presente propuesta.

2. Descripción de la Solución.- La estrategia propuesta fue desarrollar y mantener un servicio que reúna información actualizada y asegure un fácil y rápido acceso a través de una aplicación móvil para usuarios celíacos y allegados. Sumamos una página web, mediante la cual interactúan proveedores ingresando sus productos, y administradores que validan e ingresan información al sistema. La figura I muestra una vista a gran escala del sistema mencionado.

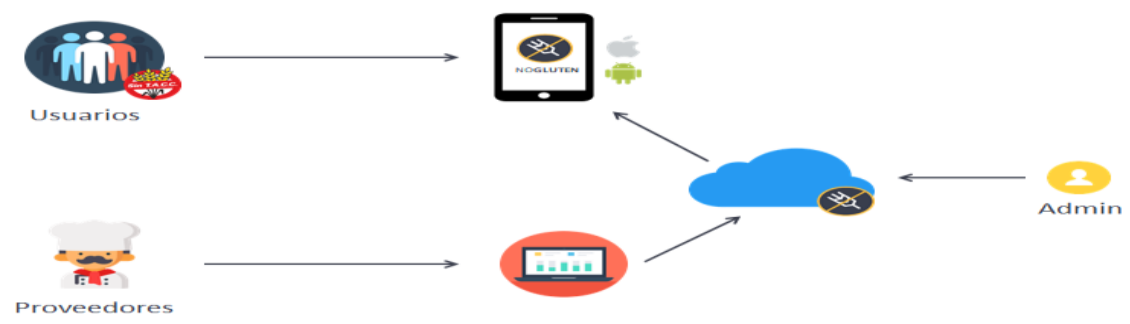

Figura I.- Diagrama general del sistema

Memoria Investigaciones en Ingeniería, núm. 18 (2020). pp 11-16

https://doi.org/10.36561/ING.18.3

ISSN 2301-1092 • ISSN (en línea) 2301-1106 
La información comprende:

- Listado de alimentos e insumos libres de gluten (aplicable por ejemplo en supermercados).

- Sitios de compra de alimentos e insumos libres de gluten (por ejemplo, góndolas especiales en supermercados o comercios que se especializan en estos productos).

- Sitios gastronómicos con menú libres de gluten (restaurantes o locales que elaboran comida; detallando si además poseen servicio de delivery).

- Recetas que sólo incluyen insumos libres de gluten.

- Tips y recomendaciones para el viajero (tanto para uruguayos que viajen al exterior como a turistas que visiten nuestro país).

- Noticias y novedades de interés.

Las fuentes de información para los ítems antes mencionados se basan en Ministerio de Salud Pública, Asociación de Celíacos del Uruguay (ACELU), Comunidad de Celíacos del Uruguay (CCU), información contenida en las etiquetas de alimentos y productos (declaración de "libre de gluten" o equivalente), información publicada por sitios gastronómicos, recomendaciones de grupos y personas celíacas, información ingresada por empresas, entre otros. Oportunamente se podrá recurrir a información de similares fuentes de otros países. La información es ingresada por los administradores del sistema a partir de la web, pero también por los propios usuarios mediante reportes, sugerencias o calificaciones, a través de la aplicación móvil. La información contribuida por usuarios es sometida a un proceso de validación por parte de los administradores.

El sistema propuesto permite la búsqueda de información sobre alimentos e insumos por distintas vías como el escaneo del código individual del producto (código de barras), búsqueda por categoría o nombre y listados libre. Los sitios de compra y locales gastronómicos por distribución en el mapa o en relación a la ubicación del dispositivo móvil. Las recetas por nombre o categoría. Los tips, recomendaciones, novedades y noticias según país, fecha de publicación y categoría. Los proveedores de productos aptos pueden registrarse e ingresar productos a través de la web, luego de ser validados por un administrador. Uno de los requerimientos esenciales para el sistema es que sea de uso sencillo, amigable e intuitivo que no requiera una etapa de entrenamiento. Se espera pueda ser utilizado por personas no altamente familiarizadas con la tecnología.

Finalmente queremos señalar que esta herramienta genera un repositorio propio a partir de información que ya está disponible (etiquetado de productos, información sobre empresas, páginas web, redes sociales, entre otros). Si bien la información existe, muchas veces se vuelve difícil de obtener cuando se la necesita. Además, las empresas proveedoras de alimentos, insumos y lugares gastronómicos tendrán un canal de comunicación único y específico con el potencial público objetivo.

Un caso especial es la cantidad de turistas que visitan nuestro país, que, dada la falta de información variada y unificada, encontrarán en esta herramienta un aliado útil donde sentirse respaldados. Cada leyenda debe contar con su equivalente en otros idiomas de forma de facilitar la comprensión, generando así una buena experiencia de usuario. Otro caso similar es no sólo el turismo interno, sino también moverse dentro de la misma ciudad.

3. Detalles del sistema.- Para el desarrollo de las aplicaciones móviles (frontend mobile), optamos por tecnologías que a partir del mismo código base, compilan a código nativo de cada plataforma. Las seleccionadas fueron NativeScript + Angular. Para el servidor (backend) y la web para administradores y proveedores (frontend web) utilizamos Ruby on Rails, explotando las enormes ventajas que posee a la hora de agilizar el desarrollo y escalar el sistema, obteniendo resultados tangibles desde etapas tempranas. 
A modo de ejemplo, mostraremos en forma esquemática las etapas por las que pasa una sugerencia para incorporarse al sistema (Figura II):

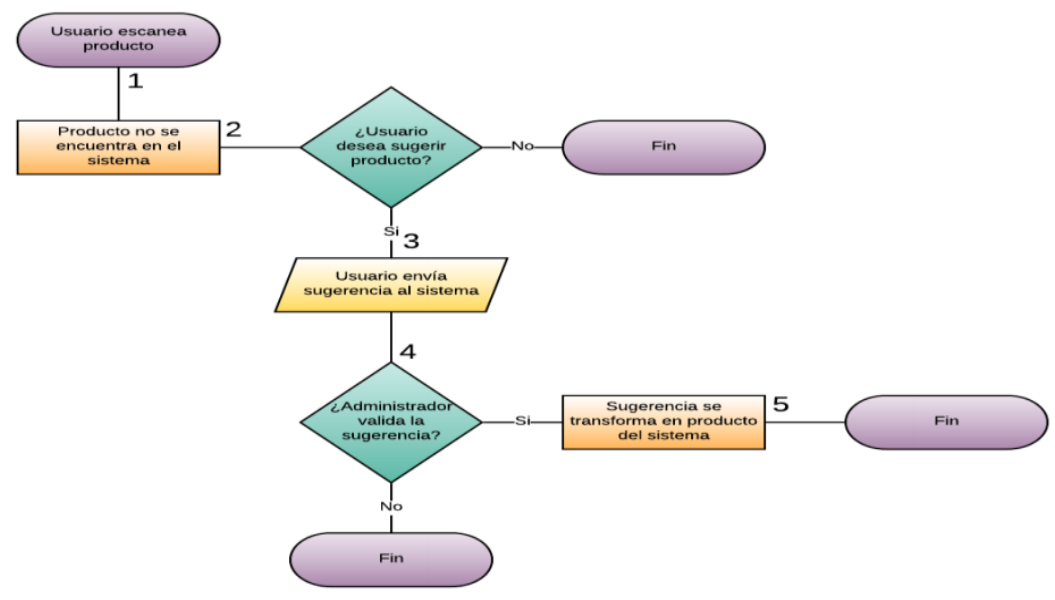

Figura II.- Diagrama de flujo de una sugerencia de producto

Las siguientes imágenes, figuras III, IV y V, son capturas del sistema en ejecución, con los pasos enumerados arriba esquematizados.
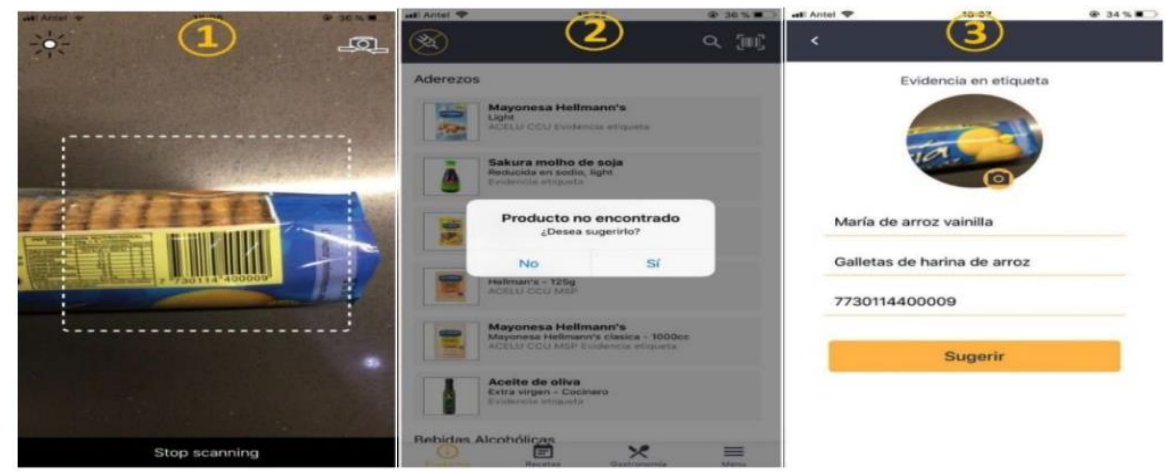

Figura III.- Secuencia de capturas pasos 1,2,3 (móvil/web)

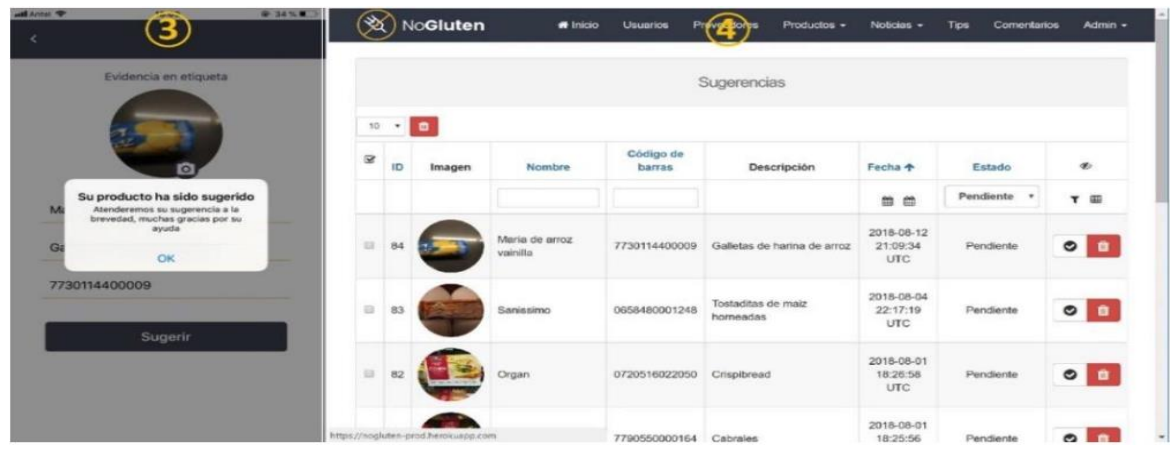

Figura IV.- Secuencia de capturas pasos 3,4 (móvil/web) 


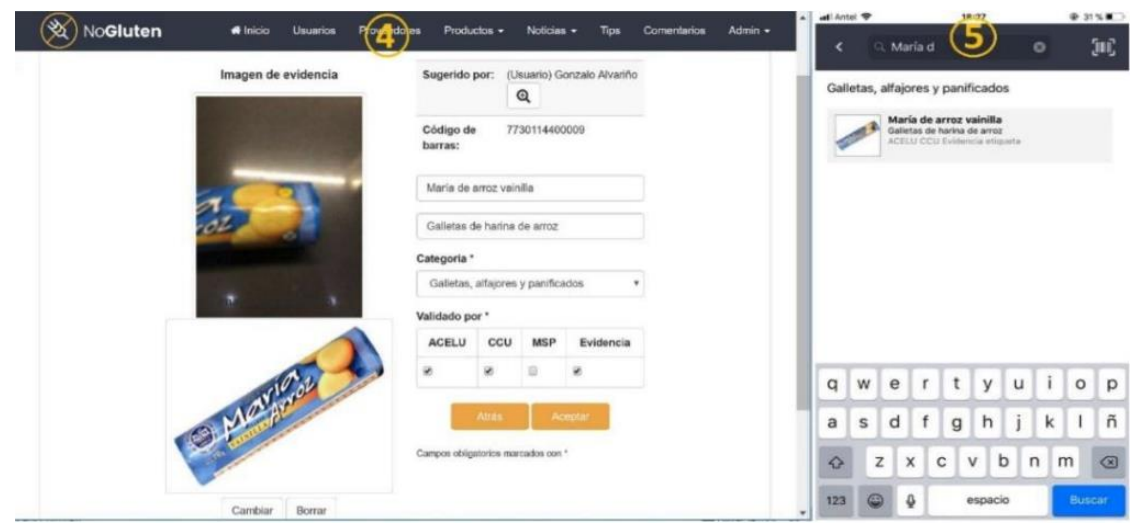

Figura V.- Secuencia de capturas pasos 4,5 (móvil/web)

Como puede visualizarse, la sugerencia de un producto conlleva un proceso de validación estricto. Desde el momento que el usuario decide llevar a cabo una sugerencia, la misma debe pasar por un filtro realizado por un administrador, pudiendo este alterar ciertos contenidos inadecuados antes de su publicación. Las validaciones de productos aptos se realizan a partir de los listados públicos ofrecidos por las asociaciones de celíacos reconocidas, Acelu y Comunidad de Celíacos del Uruguay, así como aquellos productos que posean leyendas asegurando la no prevalencia de gluten dentro de los componentes de estos.

Es importante destacar que dicho proceso fue una decisión tomada por el equipo con el objetivo de garantizar al usuario la mayor confiabilidad posible, en cada dato que es ingresado en la aplicación.

4. Conclusiones.- El presente proyecto fue el trabajo de grado de la carrera de Ingeniería en Sistemas de la Universidad ORT, año 2018. En el año 2019 fue seleccionado como Premio al Primer Puesto de Trabajos de Fin de Carrera, opción Computación, por la Academia de Ingeniería del Uruguay (ANIU) [9-10].

Los objetivos académicos pudieron ser alcanzados con éxito. Las experiencias y conocimientos adquiridos durante la carrera nos permitieron sobrepasar desafíos generados por la gestión del proyecto, así como también por las nuevas tecnologías utilizadas, destacando lo valioso de ser un equipo multidisciplinario. En cuanto al producto, desarrollamos una solución con desafíos tecnológicos donde el foco fue generar funcionalidades que aporten valor a los usuarios, satisfaciendo las necesidades detectadas, y fomentando la colaboración; basado en una arquitectura sólida que cumplió con los atributos de calidad esperados.

El sistema está iniciando su etapa operativa, centrados en cargar información para posteriormente proceder a difundir su uso. Una vez alcanzado un flujo de usuarios considerable, se implementará un modelo de negocio basado en publicidad a fin de asegurar la sustentabilidad y permanente desarrollo. Podrán también explorarse otras estrategias de orden comercial con este mismo objetivo.

Es de esperar pueda convertirse en una herramienta de uso diario por parte de la comunidad celíaca generando múltiples vínculos tanto a nivel nacional como regional en pos de asegurar el acceso a productos libre de gluten apostando a la inclusión social y la mejora de la calidad de vida. 


\section{Referencias.-}

[1] M. Rewers, "Epidemiology of celiac disease: what are the prevalence, incidence, and progression of celiac disease?," Gastroenterology, vol. 128, no. 4, 2005. [Online], Available: https://www.gastrojournal.org/article/S0016-5085(05)00199-X/pdf . [Accessed Ago. 18, 2018]

[2] M. F. Kagnoff, "Overview and pathogenesis of celiac disease," Gastroenterology, vol. 128, no. 4, 2005. [Online], Available: https://www.gastrojournal.org/article/S00165085(05)00177-0/pdf . [Accessed Ago. 18, 2018]

[3] FACE. "Qué es el gluten" [Online], Available: https://celiacos.org/enfermedadceliaca/que-es-el-gluten/. [Accessed Feb. 18, 2020]

[4] SEGHNP. "Protocolo para el diagnóstico precoz de la enfermedad celíaca" [Online], Available: https://www.seghnp.org/documentos/protocolo-para-el-diagnostico-precozde-la-enfermedad-celiaca . [Accessed Ago. 18, 2018]

[5] Ministerio de Salud Pública. "Guía de detección precoz, diagnóstico y tratamiento de la enfermedad Celíaca" [Online], Available: https://www.gub.uy/ministerio-saludpublica/comunicacion/publicaciones/guia-deteccion-precoz-diagnostico-tratamientoenfermedad-celiaca . [Accessed Ago. 18, 2018]

[6] A. Sapone, et. al., "Open Access Spectrum of gluten-related disorders," BMC Medicine, vol. 10, no. 13, 2012. [Online], Available:

https://bmcmedicine.biomedcentral.com/track/pdf/10.1186/1741-7015-10-13. [Accessed Ago. 18, 2018]

[7] Ministerio de Relaciones Exteriores de Chile. "Opiniones de agentes relevantes en el mercado sobre productos de la competencia local y extranjera" [Online], Available: https://www.prochile.gob.cl/wpcontent/uploads/2016/08/Tendencias EEUU Gluten 2016.pdf. [Accessed Ago. 18, 2018]

[8] Federación de Asociación de Celiacos de España. "El mercado mundial sin gluten sigue creciendo" [Online], Available: https://celiacos.org/mercado-mundial-sin-gluten/ . [Accessed Aug. 19, 2018]

[9] G. Alvariño, et. al., "NoGluten: Plataforma de apoyo a la celiaquía," Tesis de grado, ORT, Montevideo, 2018. [Online], Available: https://dspace.ort.edu.uy/handle/20.500.11968/3853 . [Accessed Feb. 18, 2020]

[10] Academia Nacional de Ingeniería del Uruguay. ANIU. [Online], Available: https://www.aniu.org.uy/en/home-3/. [Accessed Feb. 18, 2020] 